Arie J. Kalis • W. O. van der Knaap •

Astrid Schweizer • Ralf Urz

\title{
A three thousand year succession of plant communities on a valley bottom in the Vosges Mountains, NE France, reconstructed from fossil pollen, plant macrofossils, and modern phytosociological communities
}

Received: 24 August 2005 / Accepted: 19 January 2006 / Published online: 27 July 2006

(C) Springer-Verlag 2006

\begin{abstract}
Pollen and macrofossils were studied in a core from a fen at the foot of a slope in the Vosges Mountains, NE France. The present-day vegetation of little disturbed Abies, Fagus, and Picea forest and wetlands has been described in detail in terms of phytosociological communities using the Braun-Blanquet approach. Past ecological conditions are reconstructed in five steps: (1) The modern vegetation types are described as combinations of phytosociological species groups. (2) Micro- and macrofossils are assigned to these groups. (3) These in combination determine the past vegetation types at the site; there were simultaneously several such types in some biozones. (4) The sequence of past vegetation types is interpreted as successional pathways. (5) Past ecological conditions are inferred from these pathways. Results are: (1) The types of local forest and fen were the same around 1000 B.C. as today. (2) Rising groundwater around 650 B.C. caused a natural wet meadow to develop at the site. (3) Trees were felled near the site in the first century B.c. (Late Iron Age), facilitating the immigration of Picea. (4) Groundwater level rose during early Medieval times because of a wetter climate and alder carr replaced the dry-soil forest close to the site. (5) During High Medieval Times (10th-13th century) the nearby raised bog expanded over the site. (6) Forestry starting around A.D. 1750 caused nutrient-rich water to reach the site, resulting in abrupt vegetation change. (7) The creation of a forest road around A.D. 1855 (historical information) caused further nutrient
\end{abstract}

Communicated by W. Tinner

A. J. Kalis · A. Schweizer · R. Urz

Institut für Archäologische Wissenschaften der J.W.

Goethe-Universität, Abteilung Vor- und Frühgeschichte, Labor für Archäobotanik,

Grüneburgplatz 1,

60323 Frankfurt am Main, Germany

W. O. van der Knaap ( $₫)$

Institute of Plant Sciences, University of Bern,

Altenbergrain 21,

3013 Bern, Switzerland

e-mail: knaap@ips.unibe.ch enrichment of the site. The validity of the method used depends on the assumption that past and present vegetation types are virtually identical, which is true in our study area and study period, according to all the indications that we have.

Keywords Braun-Blanquet · Pollen · Macrofossils . Succession $\cdot$ Phytosociological species groups

\section{Introduction}

In the approach taken by the French-Swiss school of phytosociology (Braun-Blanquet 1928, 1932, 1964), presentday vegetation is seen as being structured in phytosociological communities; and the assumption is made that this was also the case in the past (Tüxen 1974a). From the reconstruction of those past communities from the fossil record, inferences can be made about the environment of the past ecosystem, thus fulfilling one of the aims of modern Quaternary palaeoecology.

Waterlogged sediments often contain large amounts of well-preserved plant remains. In situations where the sediment is largely autochthonous, most of the fossils once lived within the basin of deposition. Autochthonous deposits such as peat have the highest potential to contain plant communities fossilised nearly in their entirety. But even the record of past peat communities is usually incomplete, because not all species are preserved, recovered, and recognised. An additional complication is that even autochthonous deposits often contain fossil plant assemblages derived from several different plant communities.

The first step in reconstructing the incompletely preserved communities is to determine the taxonomic affinities of the fossils with modern communities. A number of species identified in the fossil assemblage, through their known modern niches and the ways in which they are associated, can be used to indicate the past occurrence of particular communities; this is the so-called "application 
backwards in time of known ecological and sociological preferences of taxa" (Birks and Birks 1980, p. 231). Such species with a well-defined narrow ecological tolerance are called indicator species (Janssen 1967, 1986). The use of indicator species, however, has its limitations for the following reason. In modern floristic phytosociology (according to the Braun-Blanquet approach) plant communities (phytocoenoses) are defined in terms of taxonomic (qualitative) rather than quantitative composition. The type of community is determined by the presence or absence of so-called diagnostic species (character- and differentiating species). Presence or absence of particular fossils might therefore provide a basis for reconstructing past communities. Unfortunately, diagnostic species typically have a narrow ecological amplitude, occurring in a few communities only, this being what makes them diagnostic in syntaxonomy. Diagnostic species are therefore rare in real vegetation and may be lacking in fossil assemblages. This makes them of little use for the recognition of the syntaxonomic type of most actual plant communities. The importance of diagnostic species lies in their value as exclusive species in the syntaxonomic system; they were never meant to enable the identification of a given stand of vegetation, let alone a particular fossil plant assemblage.

Because of the wide ecological range of the more abundant (not diagnostic) plant species, most plant communities combine species from several series of syntaxa. Therefore the phytosociological position of communities is determined not from the behaviour of single plant species, but by comparing the full combination of species with that of the syntaxa described in the formal syntaxonomic system; it is the combination of species rather than a few diagnostic species that characterises the plant communities and that is used to identify them in the field (Scamoni and Passarge 1959). This method differs from the Uppsala school of vegetation science in its acceptance of the association concept in the sense of Braun-Blanquet (Malmer and Trass 1973).

We therefore attempt to identify the past vegetation types by using characteristic combinations of taxa from the total list of fossils encountered in the fossil assemblages. Special care is needed, because fossil assemblages do not fulfil the requirements of homogeneity of vegetation and completeness of species inventory as set by the modern phytosociological approach of vegetation sampling (Mueller-Dombois and Ellenberg 1974; Schaminée et al. 1995), and also the information on taxon abundance and cover is lost. The fossil assemblages are incomplete as they depend on the former input of plant remains, preservation, and taphonomic processes; however they retain their characteristic taxonomic composition, provided that a reasonable number of taxa are preserved.

One method of reconstructing past vegetation that uses the complete fossil assemblage of taxa starts with the delimitation of recurrent groups of fossils (pollen, spores, and macrofossils) in temporal sequences by way of visual arrangement of the fossils in diagrams. Each recurrent group includes fossils that are consistently found together and is interpreted as having formed a community in the past. In the studies by Rybníček and Rybníčková (1974),
Große-Brauckmann (1968, 1976), and Janssen and Birks (1994a, b), these groups generally make good ecological and phytosociological sense and can be readily related to modern vegetational analogues. Whereas Janssen and Birks (1994a) strive for some numerical delimitation of recurrent groups through quantitative methods, the other authors stress the subjective arrangement of species in appropriate groups of similar ecology and phytosociology (e.g., GroßeBrauckmann 1986, p. 605). The method of recurrent groups can give detailed results only if the fossils of just one plant community are encountered in a sediment sample. Usually, however, modern wetland vegetation is a mosaic of different plant communities and this was probably also the case in previous times. Thus in sediments that contain the remains of several plant communities, the use of recurrent groups leads only to very generalised reconstructions such as alder carr, sedge swamp, wet meadow etc., as is the case in the studies cited above. Here, we propose a different, more refined approach, resulting in a syntaxonomically more detailed reconstruction.

In this paper we reconstruct past communities from fossil assemblages by using groups of plant species showing similar phytosociological behaviour today. Modern communities can be defined by the presence and absence of sociological species groups, a method first described by Scamoni and Passarge (1959). Sociological species groups are groups of species with similar phytosociological behaviour, i.e. they occur as a group in more or less the same list of plant communities and are absent in all others. Species within a group often have comparable ecological demands, but this is not necessarily so because plant communities consist of complex inter-species relationships that can cause species to grow outside their ecological optimum. Sociological species groups are therefore appropriate for an alternative description of modern plant communities. The great advantage of this approach is that all species, including the common ones, play a role in the definition of plant communities. This allows different ways of unravelling fossil plant assemblages, as was pointed out by Janssen (1967).

A prerequisite of our approach is the principle of actuality. Phytosociological species groups can only be determined in modern vegetation, whereas their application to fossil assemblages demands the assumption that the plant species involved had the same sociological behaviour throughout the time span studied. The further one goes back in time, the less likely this assumption may be, because the sociological behaviour of plants depends heavily on the species pool. As long as one deals with more or less the same flora, plants will have grown together according to the same principles as they do in modern vegetation. This can be assumed for our study area of mire and woodland in the upper montane vegetation belt of the Vosges Mountains during the last three millennia. This approach may fail in earlier periods such as the Atlantic, when the fir and beech that dominate the forests today were absent. However, according to known palaeobotanical finds mesotrophic wetland vegetation seems already to have reached its present species composition in early 
Holocene times (Tüxen 1974b). The principle of actuality also requires that the environmental factors acting in the past upon the vegetation fall within the range that these factors have today. We can safely assume that this was the case in our study area, not only with factors dependent on topography (such as microclimate and soil), but also with human impact on vegetation, which ranges from absent to low even today.

\section{Location and description of the site}

The study site is a valley-bottom peat bog $\left(48^{\circ} 02.9^{\prime} \mathrm{N}\right.$, $6^{\circ} 57.1^{\prime} \mathrm{E}$; elevation $945 \mathrm{~m}$ a.s.l.) in the central Vosges Mountains, a $120 \mathrm{~km}$ long mountain range stretching NNESSW in northeastern France (Fig. 1). The central Vosges reach altitudes of around $1200 \mathrm{~m}$, culminating in the Hohneck at $1362 \mathrm{~m}$ a.s.l. The valley of concern is the Grande Basse, a $2.5 \mathrm{~km}$ long and $900 \mathrm{~m}$ wide former glacier cirque situated about $5 \mathrm{~km}$ west of the Hohneck in the municipality of La Bresse. The lowest part of the valley bottom lies at $900 \mathrm{~m}$ a.s.l. and its steep slopes reach to about $1100 \mathrm{~m}$, even to $1180 \mathrm{~m}$ on the north-facing slope. The last glaciation has left a complex pattern of glacial and periglacial features such as boulder fields, kame terraces, and kame ridges, which make the valley almost inaccessible. The kame ridges hamper the discharge of water and, in combination with a precipitation of around $2000 \mathrm{~mm} / \mathrm{year}$, have given rise to a complex mosaic of wetlands including mires, carrs, peat bogs, and wooded mires. This has further reduced the accessibility, eventually leading to the preservation of several nowadays very rare near-natural ecosystems. The vegetation types surrounding the study site are shown in Fig. 2. The valley was first opened up in A.D. 1855 , when a gravel road was built that passes the study site at a distance of ca. $55 \mathrm{~m}$.

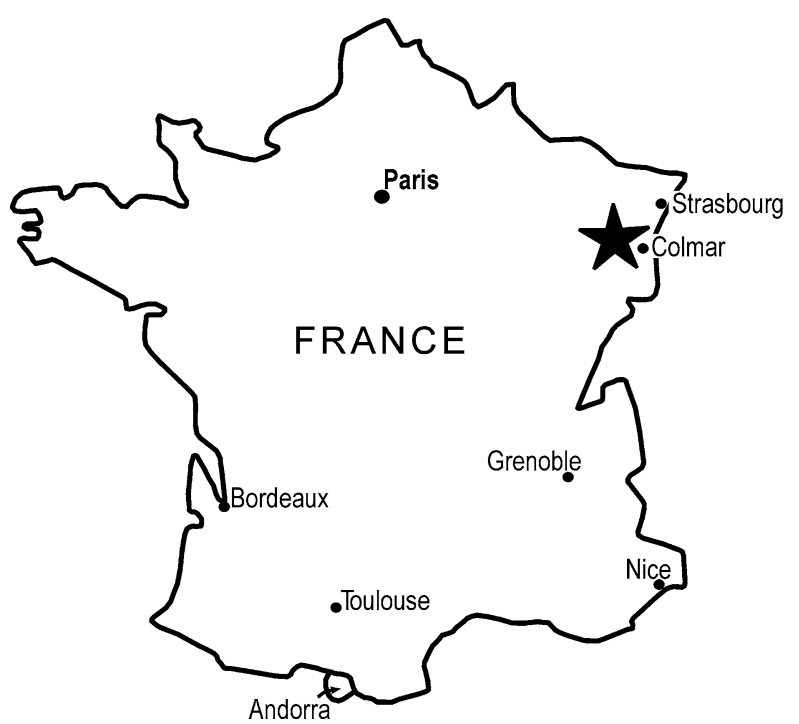

Fig. 1 A star indicates the location of the study site Grande Basse in the Vosges Mountains, France

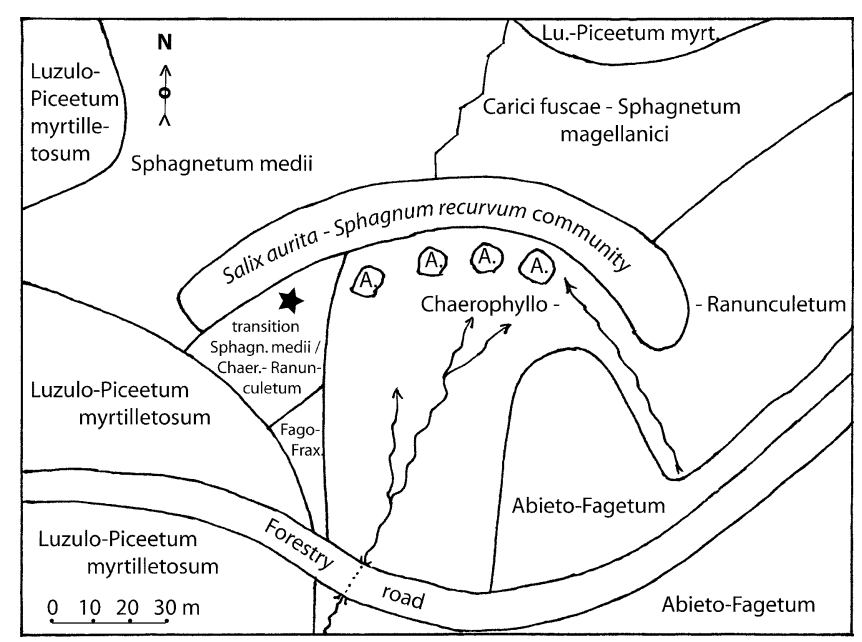

Fig. 2 Sketch map of vegetation surrounding the coring site, made in July 2005. Vegetation types are according to Table 2. The star indicates the study site; the vegetation at site is transitional between Sphagnetum medii and Chaerophyllo-Ranunculetum. A, Alnus glutinosa, small group of trees

The present vegetation of the valley is near-natural forest and mires. A diverse geology and the complex geomorphology enabled the development of a rich variety of plant communities. From a biogeographical view this valley is famous for having one of the few indigenous locations of spruce (Picea abies) in the Vosges (Kalis 1984b).

The area belongs to the upper montane vegetation belt. Fir (Abies alba) dominates the montane vegetation belt and reaches its upper limit at about $1000 \mathrm{~m}$ a.s.l., above which the woodland is dominated by beech (Fagus sylvatica) and sycamore (Acer pseudoplatanus). Spruce forests are restricted to a few steep north-facing slopes around $1050 \mathrm{~m}$ a.s.l. (plant communities belonging to Luzulo-Piceetum) and along the margins of peat bogs (Mastigobryo-Piceetum). The bedrock has a distinct impact on the plant cover. The eastern part of the valley has poor oligotrophic soils on acid granite, with coniferous forests (Luzulo-Fagetum and Luzulo-Piceetum). The western part (where the study site is) has basic granite rich in easily weathering minerals and fertile mesotrophic soils with species-rich deciduous woodland (Aceri-Fraxinetum, Fago-Fraxinetum, and Ulmo-Aceretum). Also on the wetlands the whole range from highly oligotrophic raised-bog communities to almost eutrophic megaforb (tall herb) communities is present, depending on the origin of the surface water. The relevant plant communities will be presented in more detail below.

\section{Methods \\ Pollen analysis}

Overlapping sediment cores were taken in the field using an Eijkelkamp gouge (diameter $6 \mathrm{~cm}$ ) and subsampled in the laboratory for pollen analysis with a small corer of a known volume $\left(0.3 \mathrm{~cm}^{3}\right)$. The pollen samples were 
chemically treated to extract the pollen following Fægri and Iversen (1989). The residue was mounted in silicone oil and analysed for pollen and spores. Analysis was carried out using a Leica Laborlux S microscope at magnifications from 312.5 to 787.5. Whole slides were counted.

Pollen and spore identification was made to the lowest taxonomic level using the following keys: Fægri and Iversen (1989), Punt et al. (1976-1995), and Moore et al. (1991), as well as the reference collection of the Laboratory of Archaeobotany of Frankfurt University and of the Institute of Plant Sciences of the University of Bern. The pollen types are named, and partly re-named, after plants common in the region of study, here the Vosges Mountains. The identity of the re-named pollen types is given in the caption to Fig. 3. All pollen and spore percentages are calculated on the basis of a pollen sum (100\% by definition) of trees, shrubs, and herbs growing on dry soil (shown in Fig. 3A); excluded from this sum are pollen and spores of wetland and aquatic taxa (shown in Fig. 3 parts 2 and 3).

\section{Macrofossil analysis}

Macrofossil analysis was carried out on the same core as the pollen analysis. The subsample volume was $25.5 \pm 3.6 \mathrm{ml}$. The samples were washed over a $0.2 \mathrm{~mm}$ sieve and the residue searched for plant remains. Identification of plant macrofossils was made by comparison with mod- ern reference material and the identification keys of Berggren (1969, 1981), Anderberg (1994), and KörberGrohne $(1964,1991)$. The macrofossils are depicted in the diagrams either as counts per subsample, or as presence/absence.

\section{Radiocarbon dating}

Four samples were radiocarbon dated (Table 1). The agedepth relationship was established by linear interpolation between calibrated radiocarbon dates.

\section{Establishment of the phytosociological species groups}

The vegetation of the municipality of La Bresse, in which the study area Grande Basse lies, has been the object of phytosociological studies by a research team from the University of Utrecht (Janssen and Punt 1998). These studies were carried out before the region was opened up for skiing tourism. This has resulted in three dissertations (De Valk 1981; Kalis 1984a; Bick 1985), several publications (Daniels et al. 1987; Janssen 1986; Janssen and Braber 1987; Kalis 1984b, 1985), and many research reports. The vegetation of the Grande Basse is extensively described in van Reenen (1979), Kalis (1984a), and Bick (1985). Similar studies of woodland vegetation in La Bresse are

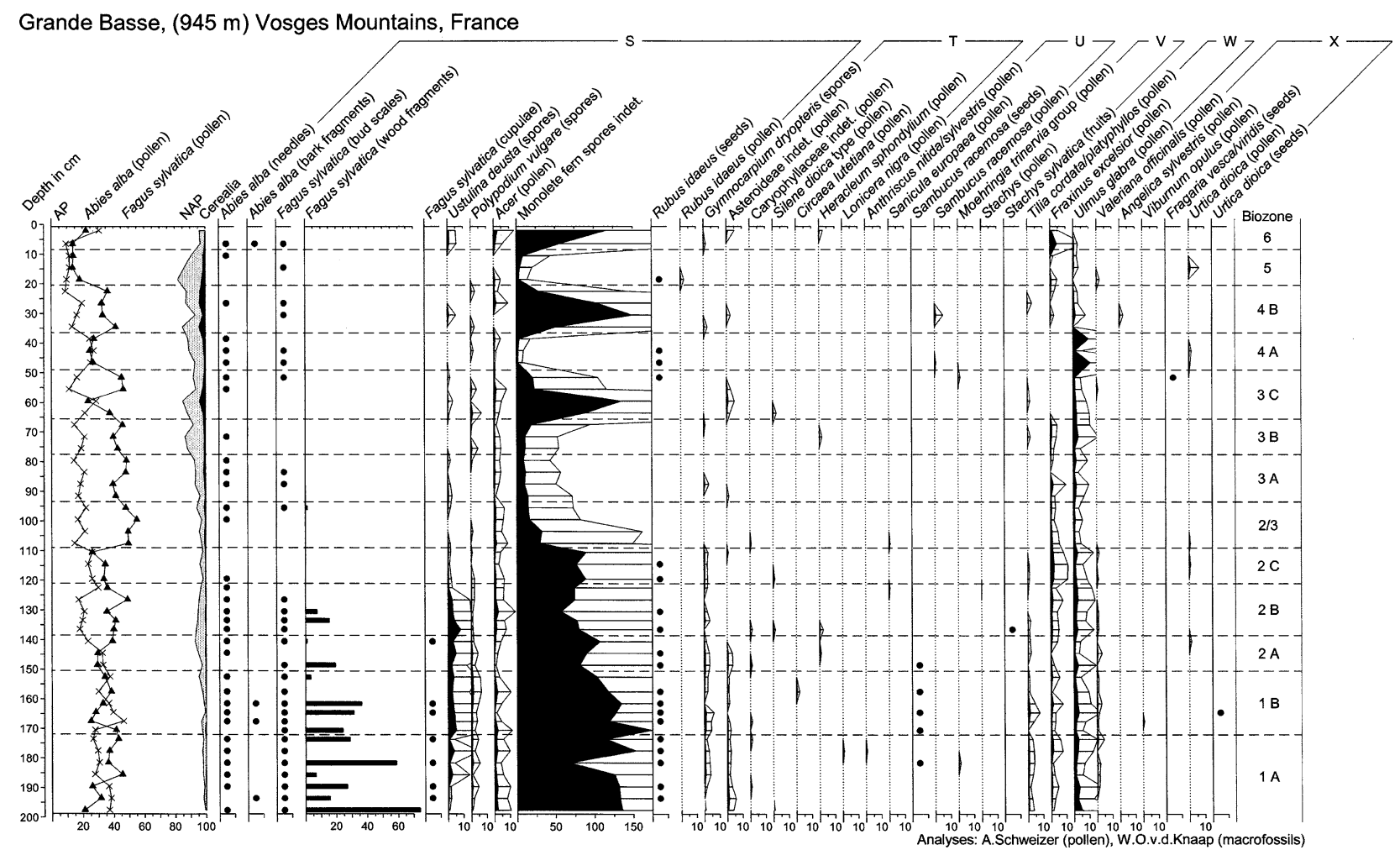

Fig. 3 Pollen and macrofossil diagram for the Grande Basse. Microfossils (pollen and spores) are drawn as black silhouettes, with 5-times exaggeration in white and depth bars at sample levels and dotted baseline; macrofossils are drawn as histograms (numbers)

or dots (presence), with solid baseline. Pollen types renamed from Punt et al. (1976-1995) are: Ranunculus aconitifolius group (Ranunculus sceleratus group), Stellaria alsine group (Cerastium fontanum group), and Stellaria nemorum group (Cerastium cerastioides group) 
by Jager (1976), Scheepstra (1978), and De Valk (1981). These studies give a comprehensive view of the modern plant communities of the Grande Basse. The delimitation of the sociological species groups used in the evaluation of the fossil plant assemblages has been made on the basis of those descriptions. Plant nomenclature follows Oberdorfer (1994).

\section{Results}

Pollen and macrofossil data

The pollen and macrofossil data are shown in Fig. 3.

Recent vegetation

Recent vegetation communities are listed in Table 2.

Phytosociological species groups

In Table 3, 97 vascular plant taxa belonging to 21 phytosociological species groups are ordered on the basis of absence/presence and dominance in the 26 vegetation types (Table 2) relevant for the study area. Five among the 97 fossil taxa encountered (pollen and/or macrofossils) are not listed for any of these vegetation types (Carex ovalis, Prunella vulgaris, Rhinanthus spec., Rumex acetosella, and Stellaria media ssp. neglecta), but the species do grow in the study area today, mostly not in closed forest but on forest tracks and along roadsides. Most of these were found as macrofossils at a depth of $51 \mathrm{~cm}$ (biozone 3C). Some of the fossils could not be assigned to species, which is regrettable notably for Poaceae and Carex because these include many relevant species. The assignment of plant species to phytosociological species groups is uncertain for some other pollen types (Asteraceae subfam. Cichorioideae). The plant communities are sufficiently well separated by the fossils, even though species not encountered as fossils are omitted. Additional information on the species groups is given below.

Group D: Carex limosa (and Scheuchzeria palustris; not found as a fossil) characterises bog hollows.

Group E: Drosera rotundifolia and Carex pauciflora (the latter not identified as a fossil) characterise raised bogs.

Group F: All species were found as fossils.

Group G: Characterised by Cyperaceae pollen; relevant species in the vegetation are mainly Eriophorum vaginatum, Carex rostrata, and Carex fusca.

Group H: Vaccinium myrtillus, V. uliginosum, and V. vitisidaea (all represented by Vaccinium pollen) have their

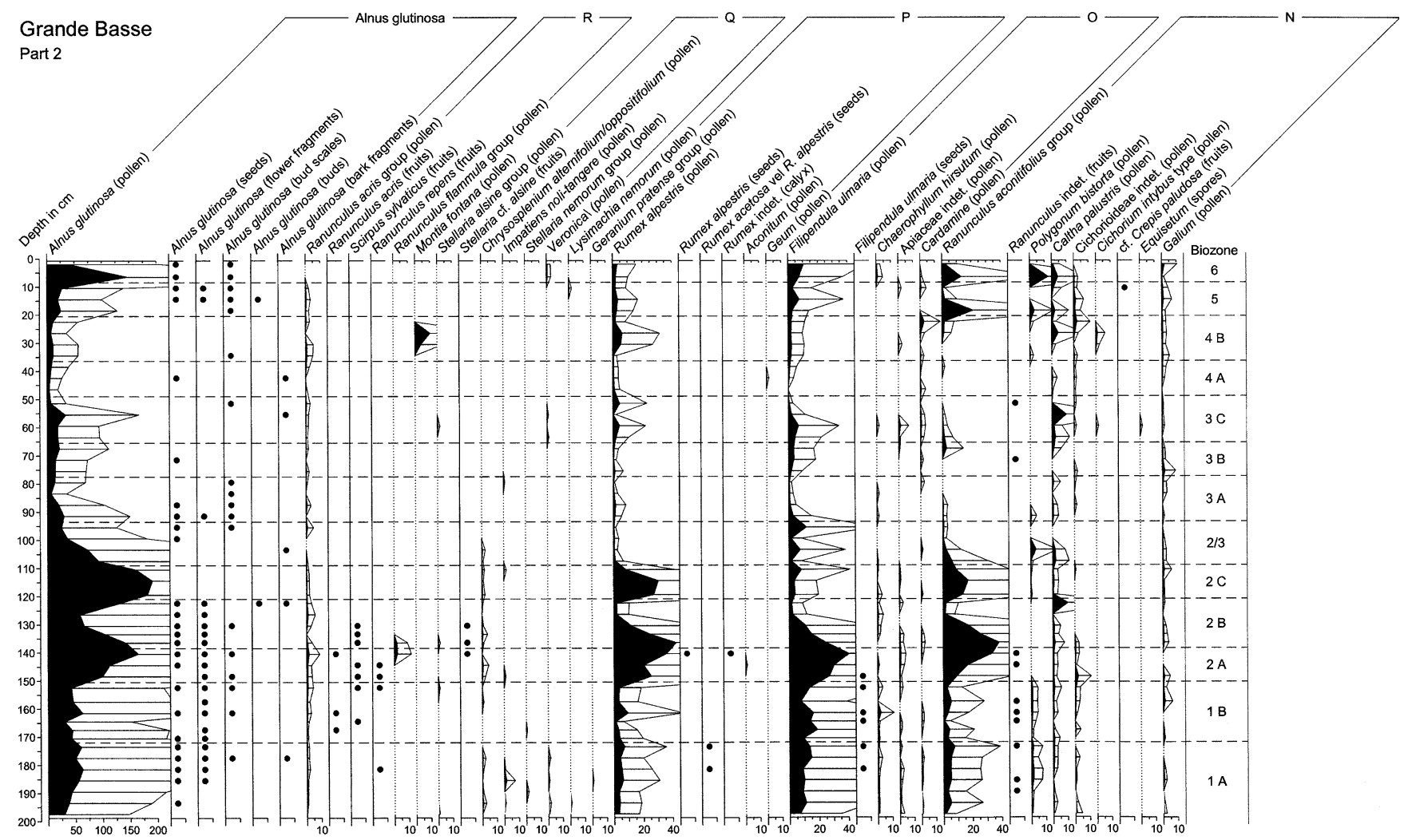

Fig. 3 Continued. 
Table 1 AMS radiocarbon dates from the Grande Basse. OXCAL 3.5 with INTCAL98 calibration data set was used for calibration $^{\mathrm{a}}$

\begin{tabular}{lllcrl}
\hline Lab. code & Depth $(\mathrm{cm})$ & Material dated & $\delta^{13} \mathrm{C}(\% \circ)$ & ${ }^{14} \mathrm{C}$ age $($ B.P. $)$ & Cal age $(1 \sigma-$ range $)$ \\
\hline Erl-4242 & 67.5 & Sphagnum stems & -29.11 & $880 \pm 55$ & A.D. $1040-1220$ \\
Erl-4311 & 127.5 & Picea needles & -28.78 & $1994 \pm 54$ & 50 B.C.-A.D. 80 \\
Erl-4243 & 135.5 & Urtica seeds & -28.85 & $1981 \pm 57$ & 50 B.C.-A.D. 80 \\
Erl-4244 & 197.5 & Fagus branch & -29.66 & $2845 \pm 64$ & 1130-910 B.C. \\
\hline
\end{tabular}

${ }^{a}$ Laboratory No.: Erl, Physikalisches Institut der Universität Erlangen-Nürnberg, Erlangen

optimum here; also important are Avenella flexuosa (Poaceae pollen) and Galium harcynicum (Galium type pollen).

Group I: Lycopodium annotinum, Huperzia selago, and Blechnum spicant characterise the transition from Picea wooded mire to Picea forest on oligotrophic mineral soil.

Group J: Relevant species are mainly Molinia coerulea, Avenella flexuosa, Agrostis canina, and to a lesser degree Deschampsia caespitosa and Festuca altissima.

Group K: Also belonging here are Carex nigra (Cyperaceae pollen) and Molinia coerulea (Poaceae pollen); Potentilla palustris and P. erecta (Potentilla pollen) have their optimum here.

Group L: Also Dactylorhiza maculata, not found as a fossil.

Group M: Salix aurita (Salix pollen) occurs at the edges of minerotrophic fens, together with Agrostis canina and Deschampsia caespitosa (Poaceae pollen), and Juncus effusus (not found as a fossil).
Group N: Fossil Galium type pollen represents mainly Galium palustre; fossil Equisetum spores represent mainly E. sylvaticum; fossil Asteraceae subfam. Cichorioideae pollen represents Crepis paludosa.

Group O: Myosotis sylvatica (not found as a fossil) grows along streams; fossil Sinapis type pollen represents Cardamine pratensis and C. amara.

Group P: Fossil Geranium pollen represents Geranium sylvaticum and G. robertianum; fossil Geum type pollen represents Geum urbanum and G. rivale; fossil Epilobium pollen represents mainly E. alpestre, E. montanum, and E. palustre.

Group Q: Fossil Chrysosplenium type pollen represents $C$. oppositifolium and $C$. alternifolium; fossil Cerastium fontanum type pollen represents mainly Stellaria nemorum and S. alsine.

Group R: Fossil Ranunculus acris-group pollen represents $R$. acris and $R$. repens.

Group S: Fossil Rubus pollen represents R. idaeus and $R$. fruticosus; belonging also to this group are Prenanthes

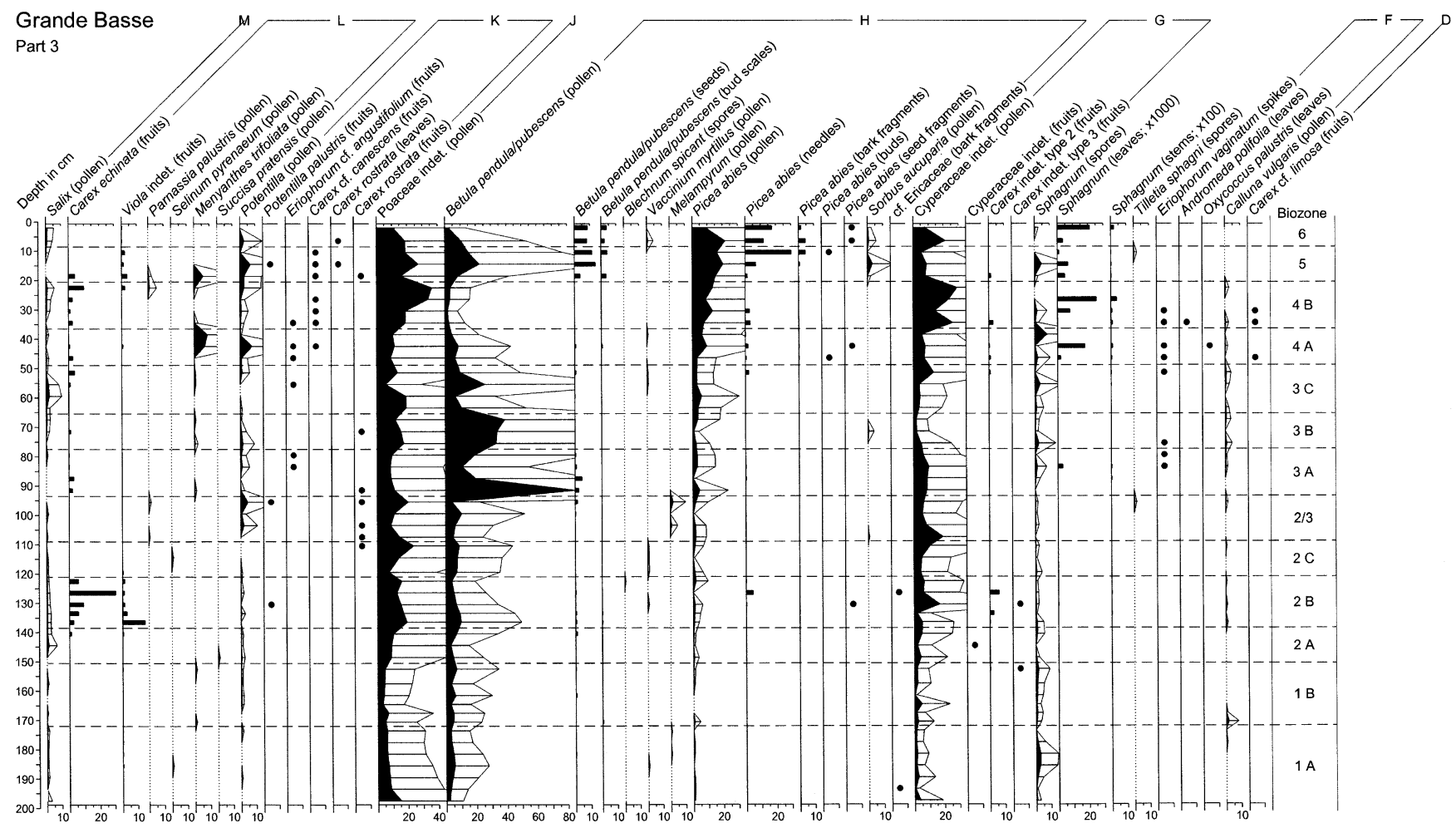

Fig. 3 Continued. 
Table 2 Plant communities described in the Vosges Mountains relevant to the fossil communities

\begin{tabular}{|c|c|c|}
\hline No. & Plant community & Based on relevées in: \\
\hline 1 & Caricetum limosae typicum & Bick (1985) \\
\hline 2 & Sphagnetum medii typicum, typical variant & Bick (1985) \\
\hline 3 & Sphagnetum medii cladonietosum arbusculae & van Reenen (1979); Bick (1985) \\
\hline 4 & Sphagnetum medii typicum, inops variant & Bick (1985) \\
\hline 5 & Sphagnetum medii typicum, Dicranum affine variant & Bick (1985) \\
\hline 6 & Carici fuscae-Sphagnetum magellanici inops, typical variant & Bick (1985) \\
\hline 7 & Pino-Sphagnetum, treeless facies & van Reenen (1979) \\
\hline 8 & Pino-Sphagnetum, treeless facies & Bick (1985) \\
\hline 9 & Initial stage of Mastigobryeto-Piceetum & van Reenen (1979) \\
\hline 10 & Pino-Sphagnetum piceetosum & Kalis (1984a); Bick (1985) \\
\hline 11 & Luzulo-Piceetum myrtilletosum & Kalis (1984a) \\
\hline 12 & Mastigobryeto-Piceetum betuletosum & Kalis (1984a) \\
\hline 13 & Betula-Sphagnum recurvum community & Bick (1985) \\
\hline 14 & Caricetum fuscae agrostietosum caninae, Potentilla palustris variant & Bick (1985) \\
\hline 15 & Caricetum fuscae agrostietosum caninae, inops variant & Bick (1985) \\
\hline 16 & Salix aurita-Sphagnum recurvum community with Vaccinium myrtillus & Bick (1985) \\
\hline 17 & Salix aurita-Sphagnum recurvum community with $S$. squarrosum & Bick (1985) \\
\hline 18 & Salix aurita-Sphagnum recurvum community & van Reenen (1979) \\
\hline 19 & Salicetum auritae & Jager (1976) \\
\hline 20 & Chaerophyllo-Ranunculetum & Scheepstra (1978) \\
\hline 21 & Chaerophyllo-Ranunculetum & De Valk (1981) \\
\hline 22 & Scirpetum sylvatici & Oberdorfer (1993) \\
\hline 23 & Chrysosplenietum oppositifolii & Jager (1976) \\
\hline 24 & Abieto-Fagetum rumicetosum & Kalis (1984a) \\
\hline 25 & Fago-Fraxinetum (Abieto-Fagetum impatientetosum) & Kalis (1984a) \\
\hline 26 & Stellario-Alnetum & De Valk (1981) \\
\hline
\end{tabular}

purpurea (Asteraceae subfam. Cichorioideae pollen), Oxalis acetosella, and Paris quadrifolia (the last two not found as fossils); fossil Acer type pollen represents mainly Acer pseudoplatanus.

Group T: Fossil Asteraceae subfam. Asteroideae pollen represents Solidago virgaurea, Adenostyles alliariae, and Senecio nemorensis. Fossil Lamium type pollen includes here only Lamiastrum galeobdolon. Also, Festuca sylvatica is represented by Poaceae pollen, and Hieracium murorum by Asteraceae subfam. Cichorioideae pollen; Myosotis sylvatica was not encountered as a fossil.

Group U: All species were also found as fossils.

Group V: Also belonging here are Carex sylvatica, represented by Cyperaceae pollen [Group G], Geranium robertianum by Geranium pollen [Group P], and Viola reichenbachiana, which was not found as a fossil. Fossil Stachys type pollen includes Stachys sylvatica, Ajuga reptans, and Galeopsis tetrahit.

Group W: All species were also found as fossils.

Group X: All species were also found as fossils.

\section{Past vegetation communities}

The left hand part of Fig. 4 shows the characterisation of the phytosociological communities occurring today in the broad surroundings of the study site (Table 2), by combi- nations of phytosociological species groups (Table 3 ). The right hand part of Fig. 4 shows the occurrence of the species groups as fossils in the biozones delimited in the pollen and macrofossil diagram (Fig. 3). This forms the basis for the reconstruction of past phytosociological communities for each biozone by means of fossils, as shown in detail in Fig. 5. Arrows in Fig. 5 indicate the potential successional pathways between the reconstructed past phytosociological communities.

\section{Interpretation}

Biozone $1 A$. Tree pollen over $98 \%$ indicates a completely forested landscape; Polypodium vulgare (an epiphyte on old deciduous trees) and Ustulina deusta (a fungus having its optimum on the bark of old Fagus trees) indicate an undisturbed state. Macrofossils of Fagus and Abies indicate that the reconstructed Abieto-Fagetum grew at the coring site. The 1-2\% Acer pollen is sufficient to indicate local presence of Acer pseudoplatanus and/or A. platanoides. Ferns dominated the forest undergrowth, as today on similar N-exposed slopes. The rather low pollen values of Fraxinus excelsior, Ulmus glabra, and Tilia (group W) indicate the presence of these trees, not in the immediate locality but in the surroundings, probably in Ulmo-Aceretum ravine-forest that still occurs today higher on the slope. Quercus, 
Table 3 Present-day phytosociological communities on the valley bottom and the lower slopes of the Grande Basse, Vosges Mountains, France $^{\mathrm{a}}$

\begin{tabular}{|c|c|c|c|c|c|c|c|c|c|c|c|c|c|c|c|c|c|c|c|c|c|c|c|c|c|c|c|}
\hline & $\begin{array}{l}\text { Phytosoc. community } \rightarrow \\
\downarrow \text { Plant taxa }\end{array}$ & 1 & 2 & 3 & 4 & 5 & 6 & 7 & 8 & 9 & 10 & 11 & 12 & 13 & 14 & 15 & 16 & 17 & 18 & 19 & 20 & 21 & 22 & 23 & 24 & 25 & 26 \\
\hline \multicolumn{28}{|c|}{ Raised bog } \\
\hline & Carex limosa & V1-2 & & & & & & & & & & & & & & & & & & & & & & & & & \\
\hline $\mathrm{I}$ & Drosera rotundifolia & II & V1 & II & III & III & II & & & & & & & & $\mathrm{R}$ & $\mathrm{I}$ & & & & & & & & & & & \\
\hline \multicolumn{28}{|c|}{ Raised and intermediate bog } \\
\hline \multirow[t]{5}{*}{$\mathrm{F}$} & \begin{tabular}{|l|} 
Andromeda polifolia \\
\end{tabular} & II & V1 & V1 & IV1 & \begin{tabular}{|l}
$\mathrm{V} 1$ \\
\end{tabular} & III & \begin{tabular}{|l|}
$\mathrm{V} 1$ \\
\end{tabular} & \begin{tabular}{|l|l|} 
III \\
\end{tabular} & I & & & & & & & & & & & & & & & & & \\
\hline & Eriophorum vaginatum & II & V1-2 & $\mathrm{V1-2}$ & V1-2 & $\mathrm{V} 2$ & $\mathrm{~V} 2$ & V2 & $\mathrm{V}+$ & III1 & II & & I & II & II & III + & $\mathrm{r}$ & & & & & & & & & & \\
\hline & Oxycoccus palustris & II & V1-2 & V1 & V1-2 & IV & V1 & V2 & V1 & III + & $\mathrm{V} 1$ & & $\mathrm{r}$ & & II & II & & & & & & & & & & & \\
\hline & Calluna vulgaris & & \begin{tabular}{|l|l}
$\mathrm{V} 2$ \\
\end{tabular} & V3 & V1-2 & V3 & IV & V2 & V2 & II & $\mathrm{V} 2$ & & II & $\mathrm{r}$ & & & & & & & & & & & & & \\
\hline & Empetrum nigrum & & & $\mathrm{r}$ & I & & I & $\mathrm{I}$ & $\mathrm{II}$ & II & III1 & & & & & & & & & & & & & & & & \\
\hline & Cyperaceae indet. & V1-2 & V1-2 & $\mathrm{V} 1-2$ & V1-2 & $\mathrm{V} 2$ & V2 & V2 & $\mathrm{V}+$ & III1 & II & & I & IV & V2 & V1 & III & III & & 1 & IV1 & & $\mathrm{V}$ & & & III + & 1 \\
\hline \multicolumn{28}{|c|}{ Mire forest } \\
\hline $\mid 1$ & Vaccinium myrtillus type & & I & II & I & $\mathrm{r}$ & $\mathrm{R}$ & V1 & V3 & V2 & $\mathrm{V} 3$ & $\mathrm{~V} 2$ & V3 & $\mathrm{V}$ & & & \begin{tabular}{|l|} 
V1 \\
\end{tabular} & I & $\mathrm{I}$ & & & & III & & & & \\
\hline & Betula pubescens (as tree) & & & & & & & $\mathrm{IV}+$ & & & $\mathrm{V} 2$ & & IV & V3 & & & V3 & III3 & & & & & & & & & \\
\hline & Melampyrum pratense & & & & $\mathrm{I}$ & & $\mathrm{r}$ & & IV1 & & II & I & & & & & & & & & & & & & & & \\
\hline & Picea abies (as tree) & & & & & & & & & & $\mathrm{V} 2$ & V1 & V3 & I & & & $\mathrm{I}$ & & & & & & & & I & II & \\
\hline & Sorbus aucuparia & & & & & & & & & & II & III & II & II & & & III & III & & & & & & & $\mathrm{I}$ & IV+ & 3 \\
\hline & Listera cordata & & & & & & & & & & $\mathrm{I}$ & & $\mathrm{r}$ & & & & & & & & & & & & & & \\
\hline & Pinus sylvestris & & & & & & & & & & III & & & & & & & & & & & & & & & & \\
\hline I & Lycopodium annotinum & & & & & & & & & & & III & & & & & & & & & & & & & & & \\
\hline & Blechnum spicant & & & & & & & & & & & III & & & & & & & & & & & & & & & \\
\hline & Huperzia selago & & & & & & & & & & & III & & & & & & & & & & & & & & & \\
\hline & Iire forest and fen & & & & & & & & & & & & & & & & & & & & & & & & & & \\
\hline $\mathrm{s}$ & Poaceae indet. & & & & & $\mathrm{I}$ & V1-2 & I & III & $\mathrm{V} 2$ & IV1 & V1 & IV1 & $\mathrm{V} 2$ & V1 & V1 & $\mathrm{V} 2$ & $\mathrm{~V} 2$ & & & IV+ & $\mathrm{V}$ & & & $\mathrm{V}$ & $\mathrm{IV}+$ & 4 \\
\hline & en & & & & & & & & & & & & & & & & & & & & & & & & & & \\
\hline & Carex rostrata & II & & & I & & II & & & & & & & III2 & V2 & V1 & III & III & & 1 & IV1 & IV & & & & & \\
\hline & \begin{tabular}{|l|} 
Potentilla type \\
\end{tabular} & & & & $\mathrm{r}$ & $\mathrm{r}$ & $\mathrm{V}+$ & & & & & & & $\mathrm{V}+$ & V2 & $\mathrm{V} 1$ & IV & III & III1 & & I & II & I & & & & 2 \\
\hline & Potentilla palustris & & & & & & & & & & & & & I & V2 & II & IV & III & III 1 & & & & & & & & \\
\hline & Viola palustris & & & & & & & & & & & & & III & V1 & IV2 & III2 & $\mathrm{V} 2$ & V 1 & & II & & & & & & \\
\hline & Carex canescens & $\mathrm{I}$ & & & & & & & & & & & & III & III & & & $\mathrm{r}$ & & & & & & & & & \\
\hline & Eriophorum angustifolium & II & & & I & I & II & & & & & & & & II & II & & & & & & & & & & & \\
\hline I & Carex echinata & & & & & & & & & & & & & & III & $\mathrm{IV}+$ & III & II & & & & & & & & & \\
\hline & Menyanthes trifoliata & & & & & & $\mathrm{r}$ & & & & & & & $\mathrm{r}$ & III & I & III3 & & & & & & & & & & \\
\hline & Selinum pyrenaeum & & & & & & & & & & & & & & III & $\mathrm{IV}+$ & & & & & & & & & & & \\
\hline & Parnassia palustris & & & & & & & & & & & & & & $\mathrm{r}$ & $\mathrm{r}$ & $\mathrm{r}$ & & & & & & & & & & \\
\hline & Succisa pratensis & & & & & & & & & & & & & & $\mathrm{r}$ & & III & & & & II & $\mathrm{I}$ & $\mathrm{I}$ & & & & \\
\hline & Salix aurita & & & & & & & & & & & & & II & & & V3 & V4 & IV & 2 & & & & & & & \\
\hline & iverine megaforbs (tall her & erbs) & & & & & & & & & & & & & & & & & & & & & & & & & \\
\hline & Polygonum bistorta & & & & & & & & & & & & & III & $\mathrm{I}$ & $\mathrm{r}$ & III & V2 & \begin{tabular}{|l|} 
V 2 \\
\end{tabular} & 1 & $\mathrm{~V}+$ & III & $\mathrm{V}$ & & I & & 3 \\
\hline & Caltha palustris & & & & & & & & & & & & & & $\mathrm{I}$ & & III & V1 & \begin{tabular}{|l|} 
V1 \\
\end{tabular} & 1 & $\mathrm{~V}+$ & IV & IV & I & & & \\
\hline & Crepis paludosa & & & & & & & & & & & & & & I & $\mathrm{r}$ & & III & IV + & 2 & IV+ & & I & I & & II & 1 \\
\hline & Ranunculus aconitifolius & & & & & & & & & & & & & II & & & $\mathrm{r}$ & IV1 & $\mathrm{V}+$ & 2 & III2 & & & & & $\mathrm{r}$ & 2 \\
\hline & Equisetum type & & & & & & & & & I & & & & & II & & II & I & V1 & 1 & IV+ & II & II & & & & \\
\hline & Rubiaceae & & & & & & & & & & & III & & II & I & & I & $\mathrm{IV}+$ & III1 & 2 & IV+ & IV & & & $\mathrm{V}$ & V1 & 1 \\
\hline & Cichorioideae indet. & & & & & & & & & & & & & & I & $\mathrm{r}$ & & III & IV+ & 2 & IV+ & III & I & I & IV & V1 & 3 \\
\hline $\mathrm{C}$ & Chaerophyllum hirsutum & & & & & & & & & & & & & & & & & $\mathrm{r}$ & $\mathrm{IV}+$ & 1 & \begin{tabular}{|l|l|} 
V2 \\
\end{tabular} & $\mathrm{V}$ & & IV2 & & & 3 \\
\hline & \begin{tabular}{|l} 
Filipendula ulmaria \\
\end{tabular} & & & & & & & & & & & & & & & & & I & & 2 & \begin{tabular}{|l|}
$\mathrm{V} 2$ \\
\end{tabular} & IV & II & II & & & 2 \\
\hline & Sinapis type & & & & & & & & & & & & & & & & & III & & 1 & V1 & IV & $\mathrm{V}$ & III & II & III & 2 \\
\hline $\mathrm{I}$ & Rumex alpestris & & & & & & & & & & & & & & & & & & & 2 & IV + & IV & & I & $\mathrm{V}$ & IV + & \\
\hline & Lysimachia nemorum & & & & & & & & & & & & & & & & & II & & 1 & IV2 & III & & & III & $\mathrm{V}+$ & \\
\hline & Primula elatior & & & & & & & & & & & & & & & & & & & & III+ & II & II & & & III + & 4 \\
\hline & Geranium type & & & & & & & & & & & & & & & & & & & 1 & $\mathrm{~V}+$ & $\mathrm{V}$ & I & & III & $\mathrm{IV}+$ & 4 \\
\hline & Epilobium indet. & & & & & & & & & & & & & & $\mathrm{I}$ & & & II & & 1 & II & III & & & III & III + & 3 \\
\hline & Aconitum napellus & & & & & & & & & & & & & & & & & & & 1 & & $\mathrm{I}$ & & & $\mathrm{I}$ & $\mathrm{I}$ & 1 \\
\hline & Geum type & & & & & & & & & & & & & & & & & & & 1 & III & & & & & I & 3 \\
\hline & tream vegetation & & & & & & & & & & & & & & & & & & & & & & & & & & \\
\hline & Impatiens noli-tangere & & & & & & & & & & & & & & & & & $\mathrm{r}$ & & 2 & $\mathrm{I}$ & & & \begin{tabular}{|l|} 
V3 \\
\end{tabular} & $\mathrm{V}$ & $\mathrm{V} 2$ & 3 \\
\hline & Chrysosplenium type & & & & & & & & & & & & & & & & & & & 2 & II & I & & V3 & II & II & \\
\hline & Cerastium fontanum type & & & & & & & & & & & & & & & & & & & 2 & I & II & & IV2 & III & V1 & 3 \\
\hline & Stellaria nemorum & & & & & & & & & & & & & & & & & & & 2 & I & $\mathrm{I}$ & & IV2 & III & V1 & 3 \\
\hline & Veronica montana & & & & & & & & & & & & & & & & & & & & I & & & II & & IV+ & \\
\hline & Stellaria alsine & & & & & & & & & & & & & & & & & & & & $\mathrm{r}$ & & & & & & 1 \\
\hline
\end{tabular}


Table 3 Continued.

\begin{tabular}{|c|c|c|c|c|c|c|c|c|c|c|c|c|c|c|c|c|c|c|c|c|c|c|c|c|c|c|c|}
\hline & \begin{tabular}{|l|} 
Phytosoc. community $\rightarrow$ \\
$\downarrow$ Plant taxa
\end{tabular} & 1 & 2 & 3 & 4 & 5 & 6 & 7 & 8 & 9 & 10 & 11 & 12 & 13 & 14 & 15 & 16 & 17 & 18 & 19 & 20 & 21 & 22 & 23 & 24 & 25 & 26 \\
\hline \multicolumn{28}{|c|}{ Wood club-rush meadow (named after Scirpus sylvaticus) } \\
\hline \multirow[t]{7}{*}{$\mathrm{R}$} & Scirpus sylvaticus & & & & & & & & & & & & & & & & & & & & & & $\mathrm{V}$ & & & & \\
\hline & \begin{tabular}{|l} 
Valeriana dioica \\
\end{tabular} & & & & & & & & & & & & & & & & $\mathrm{r}$ & & $\mathrm{r}$ & & & & $\mathrm{V}$ & & & & \\
\hline & Ranunculus acris group & & & & & & & & & & & & & & & & & & & & & & $\mathrm{V}$ & & & & 1 \\
\hline & Ranunculus repens & & & & & & & & & & & & & & & & & & & & & & III & & & & 1 \\
\hline & Ranunculus acris & & & & & & & & & & & & & & & & & & & & & & $\mathrm{V}$ & & & & \\
\hline & Ranunculus flammula & & & & & & & & & & & & & & & & & & & & & & II & & & & \\
\hline & Lychnis flos-cuculi & & & & & & & & & & & & & & & & & & & & II & & $\mathrm{V}$ & & & & \\
\hline \multicolumn{28}{|c|}{ Beech-fir mixed forest (Fagus-Abies) } \\
\hline \multirow[t]{7}{*}{$\mathrm{S}$} & Abies alba (as tree) & & & & & & & & & & $\mathrm{r}$ & $\mathrm{V} 2$ & V2 & III & & & I & & & & & I & & & III & V2 & \\
\hline & Fagus sylvatica & & & & & & & & & & & $\mathrm{V}$ & & & & & & $\mathrm{r}$ & I 1 & & & & & IV3 & $\mathrm{V}$ & $\mathrm{V} 2$ & 2 \\
\hline & Dryopteris dilatata & & & & & & & & & & & $\mathrm{V}+$ & II & II & & & II & I & & & $\mathrm{r}$ & & & & $\mathrm{V}$ & V1 & \\
\hline & Athyrium filix-femina & & & & & & & & & & & III & $\mathrm{r}$ & II & & & $\mathrm{r}$ & IV+ & & 1 & II & I & & & $\mathrm{V}$ & $\mathrm{V} 2$ & 4 \\
\hline & Rubus idaeus type & & & & & & & & & & & III & & & & & $\mathrm{r}$ & II & IV+ & & & & & & $\mathrm{V}$ & $\mathrm{IV}+$ & 3 \\
\hline & Rubus idaeus & & & & & & & & & & & III & & & & & $\mathrm{r}$ & II & IV + & & & & & & $\mathrm{V}$ & IV+ + & 2 \\
\hline & Acer type & & & & & & & & & & & II & & & & & & & IV & 1 & II & III & & II & $\mathrm{V}$ & V1 & 3 \\
\hline \multicolumn{28}{|c|}{ Mesotrophic forest } \\
\hline \multirow{7}{*}{$\mathrm{T}-\mathrm{H}$} & Dryopteris filix-mas & & & & & & & & & & & & & & & & II & & & & & & & & $\mathrm{V}$ & V 1 & 3 \\
\hline & Asteroideae indet. & & & & & & & & & & & $\mathrm{I}$ & & $\mathrm{r}$ & & & & $\mathrm{r}$ & & & & & & & $\mathrm{V}$ & $\mathrm{V} 1$ & 3 \\
\hline & 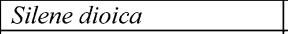 & & & & & & & & & & & & & & & & & & & & $\mathrm{I}$ & II & & & III & $\mathrm{V}+$ & 3 \\
\hline & Gymnocarpium dryopteris & & & & & & & & & & & & & & & & & & & & & & & & III & V1 & \\
\hline & Lonicera nigra & & & & & & & & & & & II & & & & & & & & & & & & & II & $\mathrm{IV}+$ & \\
\hline & Heracleum sphondylium & & & & & & & & & & & & & & & & & & & & & & & & III & $\mathrm{I}$ & 2 \\
\hline & Scrophularia nodosa & & & & & & & & & & & & & & & & & & & & & & & & II & I & \\
\hline \multicolumn{28}{|c|}{ Ravine forest (Schluchtwald) } \\
\hline \multirow{5}{*}{$\mathrm{U}$} & Ribes alpina & & & & & & & & & & & & & & & & & & & & & & & & & II & \\
\hline & Sanicula europea & & & & & & & & & & & & & & & & & & & & & & & & & II & \\
\hline & Anthriscus nitida & & & & & & & & & & & & & & & & & & & & & & & & & II & \\
\hline & Sambucus racemosa & & & & & & & & & & & & & & & & & & & & & & & & $\mathrm{I}$ & I & \\
\hline & Circaea intermedia & & & & & & & & & & & & & & & & & & & & & & & & & I & \\
\hline \multicolumn{28}{|c|}{ Forest } \\
\hline \multirow[t]{5}{*}{$\mathrm{V}$} & Anemone nemorosa & & & & & & & & & & & & & $\mathrm{r}$ & & & $\mathrm{r}$ & I & & & & & & & & $\mathrm{III}+$ & 2 \\
\hline & Moehringia trinervia & & & & & & & & & & & & & & & & & & & & & & & & & I & 4 \\
\hline & Phyteuma nigrum & & & & & & & & & & & & & & & & & & & & & & & & & II & 3 \\
\hline & \begin{tabular}{|l|} 
Stachys sylvatica \\
\end{tabular} & & & & & & & & & & & & & & & & & & & & & & & & & III+ & \\
\hline & Stachys type & & & & & & & & & & & & & & & & & & & & & & III & & & III + & 3 \\
\hline & verine forest & & & & & & & & & & & & & & & & & & & & & & & & & & \\
\hline $\mathrm{W}$ & Fraxinus excelsior & & & & & & & & & & & & & & & & & & & & & & & & & III+ & 3 \\
\hline & \begin{tabular}{|l|} 
Ulmus glabra \\
\end{tabular} & & & & & & & & & & & & & & & & & & & & & & & & I & I & 3 \\
\hline & Tilia platyphyllos & & & & & & & & & & & & & & & & & & & & & & & & II & & 3 \\
\hline & Alnus glutinosa & & & & & & & & & & & & & & & & 1 & & & & II & & & & & & 2 \\
\hline & ontane forest & & & & & & & & & & & & & & & & & & & & & & & & & & \\
\hline $\mathrm{X}$ & Corylus avellana & & & & & & & & & & & & & & & & & & & & & & & & & & 4 \\
\hline & Quercus robur & & & & & & & & & & & & & & & & & & & & & & & & & & 3 \\
\hline & Urtica dioica & & & & & & & & & & & & & & & & & & & & $\mathrm{r}$ & & & II & & & 3 \\
\hline & Angelica sylvestris & & & & & & & & & & & & & & & & & & & & & & & & & & 4 \\
\hline & \begin{tabular}{|l} 
Valeriana officinalis \\
\end{tabular} & & & & & & & & & & & & & & & & & & & & & & & & & & 2 \\
\hline & \begin{tabular}{|l} 
Fragaria vesca \\
\end{tabular} & & & & & & & & & & & & & & & & & & & & & & & & & & 2 \\
\hline & Mercurialis perennis & & & & & & & & & & & & & & & & & & & & & & & & & & 1 \\
\hline & Viburnum opulus & & & & & & & & & & & & & & & & & & & & & & & & & & 1 \\
\hline
\end{tabular}

${ }^{a}$ The phytosociological communities 1-26 are listed in Table 2. Species groups are coded D-X; species not encountered as fossils are omitted. Headers of species groups give the general vegetation types in which the groups occur. Plant nomenclature follows Oberdorfer (1994). Some species are lumped together according to their pollen morphology. Roman numerals $\mathrm{I}-\mathrm{V}$ and + and $\mathrm{r}$ indicate species frequency in present-day vegetation types; the following Arabic numerals $(1,1-2,2,3)$ are abundance/dominance according to the scale of Braun-Blanquet (1964) and Mueller-Dombois and Ellenberg (1974); 1-4 in type 26 are presence classes

Pinus sylvestris, and Carpinus betulus are absent from the nearby surroundings today and, judging by the pollen values being lower than at present, were also absent in the past. Picea abies was absent. Fossils of group Q (normally found in Chrysosplenietum oppositifolii, typical of areas with springs) suggest a spring-type forest community. The reconstructed Abieto-Fagetum impatientetosum Oberdorfer 1957 ( = Fago-Fraxinetum sensu Bartsch 1940) grows today at some hundreds of metres distance at the foot of steep $\mathrm{N}$-exposed slopes on nutrient- and base-rich soils that depend on baserich bedrock, base-rich upwelling groundwater, and a highly sheltered position (Kalis 1984a, p. 145). Local occurrence of spring water (up-welling groundwater) would also explain the presence of the reconstructed Chaerophyllo-Ranunculetum aconitifolii Oberdorfer 1952 (groups $\mathrm{N}, \mathrm{O}$, and $\mathrm{P}$ ), which is a spring-mire vegetation type on permanently percolated, base-rich, 
Fig. 4 Left: The occurrence of present-day phytosociological species groups (P.S.G.) D-X (Y-axis; Table 3) in present-day phytosociological communities 1-26 (X-axis; Table 2) in the broad surroundings of the study site. Right: The (mostly incomplete) occurrence of the same phytosociological species groups (Y-axis) as fossils in the biozones (X-axis; Fig. 2). Black field, strongly represented; gray, incompletely represented; $p$, minor representation; white, absent

Fig. 5 Reconstructed fossil plant communities; biozones are shown in the pollen and macrofossil diagram (Fig. 3). Ages B.C./A.D. are of boundaries between biozones. Fossil groups are phytosociological species groups (listed in Table 3 ) of pollen and macrofossils encountered in the biozones (Fig. 4). Combinations of groups characterise the phytosociological communities listed. Arrows indicate inferred succession pathways between plant-sociological communities (listed in Table 2); horizontal lines indicate a supposed break in the natural succession

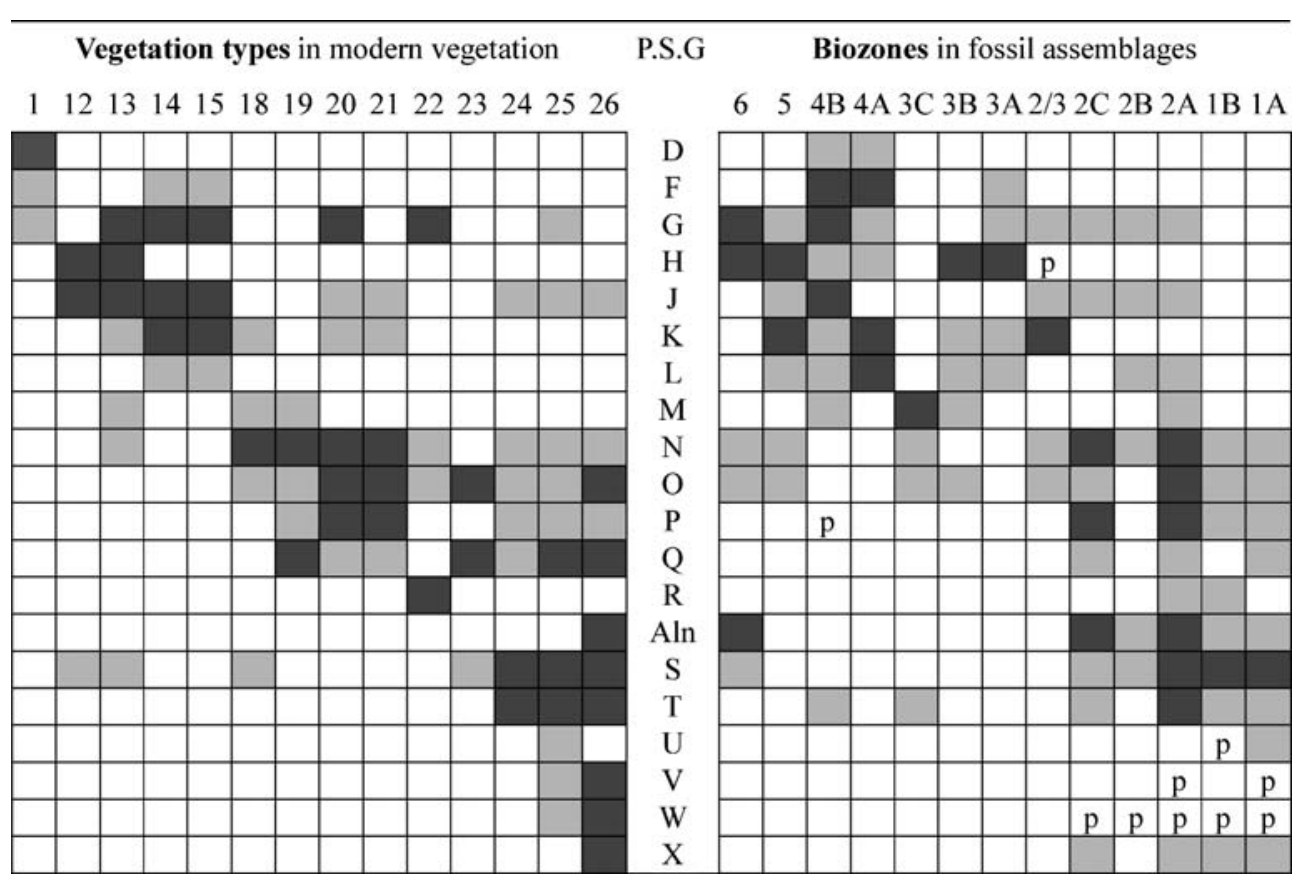

humic gley soils (Oberdorfer 1993), indicating that the spring water was emerging as an incipient stream. $\mathrm{Al}$ nus glutinosa was locally present in this vegetation type, as today, but not forming alder carr.

Biozone $1 B$. The near-disappearance of group $\mathrm{Q}$ indicates that the Abieto-Fagetum impatientetosum was no longer strictly local, though Sambucus racemosa seeds indicate that it was still close. Fossils of group $\mathrm{R}$ indicate the development of Scirpetum sylvatici, which requires a forest opening, nutrient- and baserich soil, and semi-stagnant groundwater up to the surface; it is therefore likely that the forest opening had come into being due to the local death of trees. Chaerophyllo-Ranunculetum vegetation continued on wet places where groundwater could move horizontally.

Biozone 2A. Large changes in the proportions of macrofossils (Abies and ferns declining, Alnus glutinosa, 
Rumex alpestris, Filipendula ulmaria, and Ranunculus aconitifolius increasing markedly) indicate better light conditions allowing more vigorous flowering, suggesting that the forest edge retreated from the study site. Also the area of the treeless Scirpetum sylvatici increased (group R and Cyperaceae), which indicates that the site became water-logged. An explanation for the disappearance of trees and the resulting water-logging is human impact. Although the study area is relatively inaccessible, human impact is a plausible explanation, as the southern part of the adjacent Chajoux valley (5 km distant) was intensively cultivated with fields and grasslands in this period (Late Iron Age) (Janssen and Braber 1987). The Chaerophyllo-Ranunculetum of the preceding biozone developed into an upper-montane variant of Stellario-Alnetum rich in Ranunculus aconitifolius and Rumex alpestris (De Valk 1981). Although today this is usually gallery forest along mountain streams inundated in spring, it also occurs rarely on water-logged slopes, as here in the past. De Valk (1981) described this vegetation type for the eastern Vosges, and it is known to occur below $800 \mathrm{~m}$ in the western Vosges, so its former presence here at $940 \mathrm{~m}$ altitude in the western Vosges is remarkable.

Biozone $2 B$. Re-advance of the forest edge to the study site is indicated by increased Abieto-Fagetum and the disappearance of Stellario-Alnetum and Scirpetum sylvatici. The freshly developing Caricetum fuscae agrostietosum caninae Inops-variant is a fen community on peaty soil with permanently up-welling spring water and inundation during spring. Today it is mainly found where the forest has been clear-felled, where springs initiate renewed peat formation (Bick 1985), thus strengthening the interpretation of Late Iron Age human impact as the cause of the forest opening in biozone $2 \mathrm{~A}$.

The early Picea abies needles in this biozone are of particular biogeographic interest. The indigenous as opposed to introduced status of this tree in the Vosges Mountains has long been under discussion. Kalis (1984b) showed pollen-analytically that the tree grew in the valley of the present study (Grande Basse) at least since early Medieval times. The present finding of two needles directly AMS ${ }^{14} \mathrm{C}$ dated to $1994 \pm 54$ yrs B.P. (Early Roman Age) confirms the validity of the pollen-analytical method used and even indicates an immigration date of half a millennium earlier.

The habitat preferences of Picea are relevant in deciding where the tree may first have obtained foothold. According to Kalis (1984b), the majority of natural Picea trees today (i.e. not descended from introduced trees in the area; natural and introduced Picea abies can be distinguished in the Vosges Mountains by growth form) are underdeveloped and grow on the most acid, nutrient-poor soils and on raised bogs, whereas small numbers of well-developed natural spruce trees grow in Abieto-Fagetum. In the northwestern Alps and the Jura Mountains, Picea is considered a pioneer in exploited forests, where it can establish eas- ily. Forest exploitation is thought to have caused its rapid Holocene expansion (Markgraf 1970). This was also most probably the case in our study area as the Abieto-Fagetum forests exploited by man in the Late Iron Age (biozone 2A) must have been a very suitable habitat for Picea to gain a foothold in the Vosges. However the local forest stabilised after this phase of human impact and competition with Abies prevented any further spread of spruce. Spruce growth became limited to habitats with hardly any competition from other tree species, namely on raised bogs and extremely acid soils on poor granite where natural Picea still grows today.

Biozone 2C. A renewed retreat of the forest edge from the site is shown by the lack of Fagus and Abies macrofossils, the decline of Ustulina deusta spores, and increases of Rumex alpestris, Ranunculus aconitifolius, and Alnus glutinosa pollen. The absence of Alnus glutinosa macrofossils indicates that the local Chaerophyllo-Ranunculetum wetland was too wet for the species and it grew at some distance, at the edge of the wetland.

Biozone 2/3 transition. Water-logging was the probable cause of the local disappearance of forest (AbietoFagetum). Impoverishment of the ChaerophylloRanunculetum and appearance of fen plants (group K) indicate acidification and decrease of nutrients. This suggests a shift in origin of the surface water from the nutrient-rich slopes south of the site to the raised bog northeast of the site. This shift was related to the accumulation of peat on this raised bog since the beginning of the Subatlantic estimated by Kalis (1984a) as $12.3 \mathrm{~cm}$ per 100 years on average and the resulting lateral expansion of the bog. An additional factor might have been increased precipitation during this early-Medieval time, which is also apparent from other proxy data (Schmidt and Gruhle 2003).

Biozone $3 A \pm B$. In the reconstructed Betula-Sphagnum recurvum fen community, Cyperaceae declined and $B e$ tula increased, indicating overgrowth by fen-birches (Betula pubescens ssp. carpatica Koch). Bick (1985) describes two alternative habitats for this community, minerotrophic fens below which the mineral subsoil provides sufficient nutrients to the groundwater to enable the growth of fen-birches, or along streams through the fen providing the relevant nutrients. The latter is more likely here, as a continuation of the stream-accompanying vegetation that was reconstructed for the underlying biozones.

Biozone 3C. $2 \%$ pollen of Salix (a poor pollen disperser) is sufficient to assume the local presence of willows. The fern that was so abundant is most probably Athyrium filix-femina that is at home in the reconstructed Salicetum auritae. The development to Salicetum auritae indicates a somewhat stronger contribution of nutrients in surface water, which is possibly related to a shift in the course of the stream.

Biozone 4A. The Potentilla palustris variant of the Caricetum fuscae reconstructed here develops today, ac- 
cording to Bick (1985), as secondary peat growth in stagnant parts of streams. An expansion of raised bog that is also reconstructed for this biozone is the likely cause of stagnation of the stream at the study site and the subsequent development of this community. The local development of Caricetum fuscae was therefore natural in this biozone, in contrast to the human cause assumed in biozone 2B (in which it concerned the Inops variant). The increased number of Picea macrofossils and increased spruce pollen reflect the presence of spruce trees on the approaching bog.

Biozone $4 \mathrm{~B}$. The reconstructed Caricetum limosae indicates bog-hollows, which must have developed directly from the Caricetum fuscae in the stagnant stream of the underlying biozone. This amazingly rapid acidification indicates that the laterally expanding raised bog had encroached on the site.

Biozone 5. Here we observe a break in the development, as follows. The reconstructed Betula-Sphagnum recurvum community is, as far as can be judged, identical to the present one described by Bick (1985). This, and even more so the reconstructed ChaerophylloRanunculetum, indicate an abrupt transition from extremely nutrient-poor to nutrient-rich surface water. It is clear that the nutrient-rich stream coming down the slope $\mathrm{S}$ of the site again ran through the site, as it does today, but we are unable to explain this in terms of any undisturbed development. The cause must have been man, who intensified forestry on this slope around A.D. 1750 and thus altered the hydrology and the exact courses of the streams. Although the plant communities in this part of the forest can be considered natural in their composition, their spatial distribution has been modified.

Biozone 6. The reconstructed ChaerophylloRanunculetum with Alnus glutinosa is the vegetation present at the site today. The rapid expansion of Alnus glutinosa indicates further nutrient-enrichment of the surface water, suggesting further changes in hydrology. The latter must be related to the building of the first road through the valley in A.D. 1855 (Mer 1917) that runs along the slope above the site at a distance of about $55 \mathrm{~m}$ (Fig. 1). The water formerly running down from the slope $S$ of the site over a broad front now passes under the road through pipes at a few places only, including the stream that passes the site today. This stream is therefore increased in discharge and brings much erosional material, building up a small alluvial fan between the road and the site. The limit of raised-bog vegetation is today about $20 \mathrm{~m} \mathrm{NW}$ of the site.

\section{Discussion}

Two different methods are encountered in the literature to exploit the rich palaeoecological potential of the plant micro- and macro-fossils preserved in water-logged sedi- ments. One method searches for recurrent groups of fossils and then infers their ecological indication by analogy with present-day vegetation. This method does not take into account the possibility that not one but various different vegetation types contributed to a fossil assemblage, and therefore in the best case leads to very generalised reconstructions of past vegetation (on the level of e.g. alliance, such as Alnion), or else to the reconstruction of the wrong or even non-existent vegetation types. The other method is used mainly in archaeobotany and exploits Ellenberg's ecological indicator values (Ellenberg et al. 1992) for the individual species encountered as fossils, these values being then averaged to estimate the former ecological conditions $(\mathrm{pH}$, trophic state, humidity, etc.) on the site. This method, like the preceding one, ignores the possibility of multiple vegetation types contributing to the fossil assemblage, which may lead to the reconstruction of ecological conditions of non-existent habitats. In addition, it ignores the fact that only a (mostly small) selection of the plant species that grew in the past can be retrieved as fossils, whereas Ellenberg et al. (1992) explicitly state that the calculation of ecological conditions from ecological indicator values is only valid for complete plant communities. Therefore, an assemblage of fossils that happens not to be representative in ecological terms will result in an erroneous reconstruction of ecological conditions. The method used in this paper endeavours to solve these problems by grouping the fossil assemblage into phytosociological species groups, which form the basis of the reconstruction of (potentially) multiple vegetation types from each fossil assemblage. The validity of the method depends heavily on the principle of actuality: it works only if the assumption is correct that the vegetation types reconstructed for the past occur today somewhere in the study area with virtually the same species composition. This assumption is correct for our study area according to all the indications that we have. The method requires a thorough knowledge of presentday phytosociological communities including the less common ones. This knowledge is available for our study area.

In summary, our method works as follows. (1) The microand macro-fossils encountered are assigned to phytosociological species groups determined from the present-day vegetation of the area. (2) Relying on the principle of actuality, it is assumed that the phytosociological species groups represented by fossils in an assemblage (i.e., a biozone) were growing in the past vegetation in their entirety, including the species not encountered as fossils. (3) Combinations of the reconstructed phytosociological species groups are used to infer the former presence of one or several phytosociological communities during the period represented by the fossil assemblage. (It should be noted here that the use of phytosociological species groups in identifying present-day phytosociological communities is an accepted alternative to the use of diagnostic species; see Schaminée et al. 1995). (4) The present-day ecological requirements of the reconstructed past phytosociological communities are used to infer past vegetation-successional pathways and the evolution of past ecological conditions. With this method we recon- 
structed the following environmental history of our study area:

1. The forest type growing at the site about 1000 в.C. is the same as that growing on the nearby slopes today, and the local stream-accompanying vegetation was the same as it is today.

2. At about 650 B.c. a rising groundwater table had caused a natural wet meadow at the site.

3. In the first century B.C., a period of dense habitation in nearby valleys (Late Iron Age), trees were felled in the close surroundings of the site. This short-lived phase of minor disturbance of the forest facilitated the immigration of Picea, which became established when the forest recovered in the subsequent Roman Era.

4. Groundwater level increased markedly during early Medieval Times, probably due to a cooler and wetter climate, and carr typical of wet oligotrophic soils replaced forest typical of drier nutrient-rich soils.

5. During High Medieval Times (10th-13th century) the nearby raised bog expanded laterally over the site.

6. Around A.D. 1750 forest exploitation started, disturbing the hydrology and causing nutrient-rich water to pass over the site. The vegetation changed abruptly.

7. Around A.D. 1855 a forest road was constructed (historical information) running $55 \mathrm{~m}$ from the site on the slope above, causing the stream to pass across the site resulting in a nutrient-rich stream-side vegetation. This is the situation today.

Some important aspects of the reconstruction of the past environment depend completely on the reconstruction of past phytosociological communities and their successional pathways. These are the minor human disturbance of the local forest in the Late Iron Age, the impact of climate on the local vegetation in early Medieval Times, the start of forestry around A.D. 1750, and the impact of road building in A.D. 1855 on the local hydrology.

Acknowledgements We dedicate this paper to Brigitta Ammann. Pim (W.O.) van der Knaap is especially grateful for her continual support of the scientific careers of many people of all ages and a variety of specialisations.

We thank C.R. Janssen and especially H.J.B. Birks for their perceptive reviews. This study is part of the European Union project FOSSILVA (Dynamics of forest tree biodiversity: linking genetic, Palaeogenetic and plant historical approaches; EVK2-CT-99-00036). The Institute of Plant Sciences, Bern, Switzerland provided an additional grant for pollen analysis.

\section{References}

Anderberg A-L (1994) Atlas of seeds and small fruits of NorthwestEuropean plant species with morphological descriptions 4. Resedaceae-Umbelliferae. Swedish Museum of Natural History, Stockholm

Berggren G (1969) Atlas of seeds and small fruits of NorthwestEuropean plant species with morphological descriptions 2. Cyperaceae. Swedish Natural Science Research Council, Stockholm
Berggren G (1981) Atlas of seeds and small fruits of NorthwestEuropean plant species with morphological descriptions 3. Salicaceae-Cruciferae. Swedish Museum of Natural History, Stockholm

Bick H (1985) Die Moorvegetation der zentralen Hochvogesen. Diss Bot 91, Cramer, Stuttgart

Birks HJB, Birks HH (1980) Quaternary palaeoecology. Edward Arnold, London

Braun-Blanquet J (1928) Pflanzensoziologie. Grundzüge der Vegetationskunde. Biologische Studienbücher 7. Springer, Berlin

Braun-Blanquet J (1932) Plant sociology; the study of plant communities. McGraw-Hill, New York, London

Braun-Blanquet J (1964) Pflanzensoziologie. Grundzüge der Vegetationskunde. 3rd edn. Springer, Berlin, Wien, New York

Daniels FJA, Bobbink R, Braber FI, Schild R (1987) The present and past grassland vegetation of the Chajoux and Moselotte valleys (Vosges, France) 1. The present day dwarf shrub and grassland communities. Proceedings of the Koninklijke Nederlandse Akademie van Wetenschappen C 90(2):87-114

De Valk EJ (1981) Late Holocene and Present Vegetation of the Kastelberg (Vosges, France). PhD Thesis, University of Utrecht

Ellenberg H, Weber HE, Düll R, Wirth V, Werner W, Paulissen D (1992) Zeigerwerte von Pflanzen in Mitteleuropa. Scripta Geobot 18

Fægri K, Iversen J (1989) Textbook of pollen analysis, 4th edn. Wiley, Chichester

Große-Brauckmann G (1968) Einige Ergebnisse einer vegetationskundlichen Auswertung botanischer Torfuntersuchungen, besonders im Hinblick auf Sukzessionsfragen. Acta Botanica Neerlandica 17:59-69

Große-Brauckmann G (1976) Zum Verlauf der Verlandung bei einem eutrophen Flachsee (nach quartärbotanischen Untersuchungen am Steinhuder See) II. Die Sukzessionen, ihr Ablauf und ihre Bedingungen. Flora 165:415-455

Große-Brauckmann G (1986) Analysis of vegetative plant macrofossils. In: Berglund BE (ed) Handbook of Holocene palaeoecology and palaeohydrology. Wiley, Chichester, pp 591-618

Jager ME (1976) Onderzoek naar bron- en beekbegeleidende vegetaties in de bossen ten Westen van de Grande Crêtes, Hoge Vogezen, Frankrijk. Werkgroep Vogezen Research Report 23A. University of Utrecht

Janssen CR (1967) Stevens Pond: A postglacial pollen diagram from a small typha swamp in northwestern Minnesota, interpreted from pollen indicators and surface samples. Ecol Monogr 37:145-172

Janssen CR (1986) The use of local pollen indicators and of the contrast between regional and local pollen values in the assessment of the human impact on vegetation. In: Behre K-E (ed) Anthropogenic indicators in pollen diagrams. Balkema, Rotterdam, pp 203-208

Janssen CR, Birks HJB (1994a) Recurrent groups of pollen types in time. Rev Palaeobot Palynol 82:165-173

Janssen CR, Birks HJB (1994b) Examples of recurrent groups of pollen and macrofossils in space and time delimited by simple discriminant functions. In: Lotter AF, Ammann B (eds) Festschrift Gerhard Lang - Beiträge zur Systematik und Evolution, Floristik und Geobotanik, Vegetationsgeschichte und Paläoökologie. Diss Bot 234, Cramer, Stuttgart, pp 337-351

Janssen CR, Braber FI (1987) The present and past grassland vegetation of the Chajoux and Moselotte valleys (Vosges, France) 2. Dynamic aspects and origin of grassland vegetation in the Chajoux valley, interpreted from the contrast between regional and local pollen deposition of dominant pollen types and the distribution of pollen indicators in pollen diagrams. Proceedings of the Koninklijke Nederlandse Akademie van Wetenschappen, C 90(2):115-138

Janssen CR, Punt W (1998) Fourty years pollen morphology and terrestrial Quaternary palaeobotany and palynology 1959-1998. LPP Contributions Series 11. LPP Foundation, Utrecht

Kalis AJ (1984a) Fôret de La Bresse. Vegetatiekundige en pollenanalytische onderzoekingen naar de bosgeschiedenis van een centraal-europees middelgebergte. $\mathrm{PhD}$ Thesis, University of Utrecht 
Kalis AJ (1984b) L'indigénat de l'épicéa dans les Hautes Vosges. Revue de Paléobiologie, Volume spéciale, pp 103-115

Kalis AJ (1985) La répartition des groupements forestiers sur une montagne à partir d'un exemple dans les Hautes-Vosges. Colloques Phytosociologiques 13:279-291

Körber-Grohne U (1964) Bestimmungsschlüssel für subfossile Juncus-Samen und Gramineen-Früchte. Probleme der Küstenforschung im südlichen Nordseegebiet 7, Lax, Hildesheim

Körber-Grohne U (1991) Bestimmungsschlüssel für subfossile Gramineen-Früchte. Probleme der Küstenforschung im südlichen Nordseegebiet 18:169-234

Malmer N, Trass H (1973) North European approaches to classification. In: Whittaker RH (ed) Ordination and classification of communities. Handbook of Vegetation Science 5. Junk, The Hague, pp 529-574

Markgraf V (1970) Palaeohistory of the Spruce in Switzerland. Nature 228:249-251

Mer E (1917) Association de l'Epicéa au Sapin dans les sapinières des Hautes-Vosges. Revue des Eaux et Forêts 55:262-270, 56:289295, 321-325, 353-359

Mueller-Dombois D, Ellenberg H (1974) Aims and methods of vegetation ecology. Wiley, New York

Moore PD, Webb JA, Collinson ME (1991) Pollen analysis, 2nd edn. Blackwell, Oxford

Oberdorfer E (1993) Süddeutsche Pflanzengesellschaften III. Wirtschaftswiesen und Unkrautgesellschaften. 3rd edn. Fischer, Jena

Oberdorfer E (1994) Pflanzensoziologische Exkursionsführer für Deutschland und angrenzende Gebiete. Ulmer, Stuttgart
Punt W et al. (ed) (1976-1995) Northwest European Pollen Flora, vol 1 (1976), vol 2 (1980), vol 3 (1981), vol 4 (1984), vol 5 (1988), vol 6 (1991), vol 7 (1995). Elsevier, Amsterdam

Reenen GBA van (1979) Een overgang tussen een ombrotroof groeiend hoogveen en een montaan hellingbos op relatief voedselrijk gesteente, Grande Basse, Vogezen, Frankrijk. Werkgroep Vogezen Research Report, 45. University of Utrecht

Rybníček K, Rybníčková E (1974) The origin and development of waterlogged meadows in the central part of the Šmava Foothills. Folia Geobotanica et Phytotaxonomica 9:45-70

Scamoni A, Passarge H (1959) Gedanken zu einer natürlichen Ordnung der Waldgesellschaften. Archiv für das Forstwesen 8:386426

Schaminée JHJ, Stortelder AHF, Westhoff V (1995) De vegetatie van Nederland 1. Inleiding tot de plantensociologie - grondslagen, methoden en toepassingen. Opulus, Uppsala

Scheepstra D (1978) Onderzoek naar bron- en beekbegeleidende vegetaties in de bossen ten Westen van de Grande Crêtes, Hoge Vogezen, Frankrijk. Werkgroep Vogezen Research Report 23B. University of Utrecht

Schmidt B, Gruhle W (2003) Klimaextreme in römischer Zeit. Eine Strukturanalyse dendrochronologischer Daten. Archäologisches Korrespondenzblatt 33:221-226

Tüxen R (1974a) Großreste von Pflanzen, Pollen. Sporen und Bodenprofile in ihrer Bedeutung für Syndynamik und Synchronologie. In: Knapp R (ed) Handbook of vegetation science 8. Vegetation dynamics, pp 25-42

Tüxen R (1974b) Synchronologie einzelner Vegetationseinheiten in Europa. In: Knapp R (ed) Handbook of Vegetation Science 8. Vegetation dynamics, pp 265-292 